\title{
Планирование Процессов Вычислений в Распределенных Системах
}

\author{
Джаваншир Кязимов \\ Бакинский Государственный Университет, Баку, Азербайджан \\ dkazimov@mail.ru
}

\begin{abstract}
Аннотация- Рассматриваемая работа посвящена построению оптимальной последовательности выполнения операций в заданные сроки в распределенных средах, используя функции штрафа. Для этого задается алгоритм определения оптимальной последовательности выполнения заданий.
\end{abstract}

Ключевые слова- функция штрафа, планирование процессов, оптимальное расписание

\section{I. ВВЕДЕНИЕ}

Известно, что основная функция систем управления ресурсами -это распределение программных приложений по процессорным узлам или компьютерам [1]. Цели, которые при этом могут преследоваться, являются увеличением реальной производительности, балансировкой нагрузки процессоров и т.д.

В программном обеспечении соответствующих систем можно выделить два компонента - менеджер ресурсов и планировщик. Менеджер отвечает за распределение вычислительных ресурсов, их аутентификацию, создание и миграцию процессов. Планировщик определяет очередность выполнения работ и их назначение на те или иные ресурсы.

В данной работе, используя модели составления расписаний и учитывая директивные сроки, рассматривается планирование процессов вычислений без прерываний в распределенных системах. Также рассматривается составление статистического расписания. Это означает, что планирование осуществляется до начала вычислений.

\section{II. ПОСТРОЕНИЕ ОПТИМАЛЬНОЙ ПОСЛЕДОВАТЕЛЬНОСТИ ВЫПОЛНЕНИЯ ОПЕРАЦИИ}

Пусть задается $U=\left\{u_{1}, u_{2}, \ldots, u_{n}\right\}$ множество операций, которые необходимо выполнить в распределенных системах. На множестве операций определяется отношение $\prec$ предшествование.

Пусть $s_{i}, f_{i}$ моменты начала и окончания операций $u_{i} \in U, \quad i=1,2, \ldots, n$ и для множества $U$ операций, связанных отношением предшествования, задан отрезок планирования $[0, T]$. Тогда в общем случае расписание $s h$ определяется как множество [1]:

$$
s h=\left\{\left(s_{i}, f_{i}\right)\right\}, \quad i=1,2, \ldots, n, \quad s_{i}, f_{i} \in[0, T] \text { Обозначим }
$$
через $\tau_{i}$ - длительность $i$-ой операции в расписании $s h$, а $d_{i} \geq 0$ директивные (крайние) сроки времени завершения выполнения операций $i=\overline{1, n}$. Не нарушая общности, пронумеруем все операции в порядке возрастания $d_{i}$. Выполнение операций может быть завершено в заданные сроки и $\Omega=\{1,2, \ldots, n\}$ оптимальная последовательность выполнения операций. Если хотя бы при одном значении $i$ значение $t_{i}>d_{i}$, то не существуют последовательности выполнения операций ,при которых эти операции выполнялись бы в заданные сроки. В этом случае значения функции штрафа положительны и построение оптимальной последовательности сопряжено с определенными вычислительными затруднениями.

В отличии от [2], в рассматриваемой работе для построения оптимальной последовательности выполнения операций, функция штрафа определяется следующим образом:

$$
\Phi_{i}(t)= \begin{cases}0, & \text { если } t \leq d_{i} \\ \max \left(s_{i}-\tau_{i}-d_{i}\right), & \text { если } t>d_{i}\end{cases}
$$

Качество расписания sh определяется вектором $T$, т.е. каждому расписанию sh ставится в соответствие значение некоторой скалярной функции $F(T)$ вектора $T$, определяемого расписанием sh. В качестве $F(T)$ выбираем функции:

$$
F(T)=\sum_{i=1}^{n} \Phi_{i}(t)
$$

Допустим, что имеется способ, посредством которого из множества $U=\left\{u_{1}, u_{2}, \ldots, u_{n}\right\}$ всех операций может быть выделено подмножество $B$, таким образом, что если $k \in B$, то операции $k$ в срок не выполняются. Учитывая (1) можно получить:

$$
F(T)=\sum_{i \in B} \Phi_{i}(t)=\sum_{i \in B} \max \left(s_{i}+\tau_{i}-d_{i}\right)
$$


Расписание, которое удовлетворяет всем условиям рассматриваемой задачи, называется оптимальным, если ему соответствует значение наименьшее $F(T)$.

Покажем, что в этом случае и построение самой оптимальной последовательности не вызывает затруднений, Действительно, пусть $\Omega_{B}$ произвольная последовательность элементов множества $B$, а $\Omega_{A}$ упорядоченная по возрастанию последовательность элементов множества $A=U \backslash B$. Будем выполнять операции в последовательности $\Omega=\left(\Omega_{A}, \Omega_{B}\right)$.

Учитывая тот факт, что все операции пронумерованы в порядке возрастания значений $d_{i}$. Поэтому операции множества из $A$ будут выполняться в срок. Что касается заданий множества $B$, то ни одно из них при этой последовательности не будет выполнено в срок, так как в противном случае исходная последовательность не будет оптимальной. Следовательно,

$$
F\left(\Omega_{A}, \Omega_{B}\right)=F\left(\Omega_{B}\right) \text { и } \Omega=\left(\Omega_{A}, \Omega_{B}\right)
$$

оптимальная последовательность.

\section{ЗАКЛЮЧЕНИЕ}

Используя модели составления расписаний и учитывая директивные сроки для планирования процессов вычислений без прерываний в распределенных системах, можно построить оптимальную последовательность выполнения операций.

\section{ЛИТЕРАТУРА}

[1] В.В.Топорков, Модели распределенных вычислений. М:.Физмат лит., 2004, 313 с

[2] Д.К.Кязимов, Построение оптимального упорядочения для выполнения потока заданий в заданные сроки в вычислительной системе. J. of contemporary Applied Mathematics. V.5., №1, 2015, стр.22-27. 